\title{
Photochemical Decomposition of Nalidixic Acid in Water
}

\author{
Ki-Min Bark, ${ }^{\dagger}$ Yong-Sung Kim, Chul-Ho Park, ${ }^{\dagger}$ Hyeong-Chul Lee, ${ }^{\S}$ and Hyoung-Ryun Park ${ }^{*}$ \\ Department of Chemistry, Chonnam National University, Gwangju 500-757, Korea. *E-mail: hrpark@chonnam.ac.kr \\ ${ }^{\dagger}$ Department of Chemical Education and Research Institute of Natural Science, Gyeongsang National University, \\ Chinju 660-701, Korea \\ \$Department of Health and Environment, Chunnam Techno College, Koksung 516-911, Korea \\ ${ }^{\S}$ Department of Food and Nutrition, Songwon College, Gwangju 502-742, Korea \\ Received July 29, 2005
}

\begin{abstract}
Key Words : Photochemical reaction, Nalidixic acid, 7-Methyl-4-oxo-2,4-dihydro-1H-8,8b-diaza-cyclobuta[a]naphthalene-3-carboxylic acid, 7-Methyl-4-oxo-1,4-dihydro-[1,8]naphthyridine-3-carboxylic acid, 1,7Dimethyl-4-oxo-1,4-dihydro-[1,8]naphthyridine-3-carboxylic acid
\end{abstract}

Nalidixic acid (1-Ethyl-1,4-dihydro-7-methyl-4-oxo-1,8naphthyridine-3-carboxylic acid, NAL), which was first synthesized by Lesher et al. in $1962,{ }^{1}$ is a quinolone derivatives with antibacterial properties. Many quinolone derivatives have been synthesized for clinical use. However, these drugs have been associated with phototoxic and photoallergenic reactions in human skin by sunlight. ${ }^{2-4}$ The photochemical reaction is useful for understanding these photosensitive reactions because it can produce photoproducts during the UV-irradiation process. Moreover, the pharmaceutical drugs are usually released into the environment after clinical use and pollute the natural water. The drugs dissolved in water lead not only to harmful effects on human health but also to atmospheric pollution as a result of their photochemical decomposition. Therefore, it is of interest to examine the photochemical reaction of nalidixic acid. However, there are few reports on its photochemical properties. Dezer et al. ${ }^{5}$ have reported that 1-ethyl-1,4hydro-7-methyl-4-oxo-1,8-naphthyridine (EMDN) was produced as a major product after irradiating NAL in a basic solution. There are few papers dealing with the photochemical properties of NAL ${ }^{6-7}$ as well as with the photosensitizing properties of EMDN. ${ }^{8}$ Recently, the photochemical reaction of methanolic NAL and the photochemical reaction pathway of methanolic NAL using 300 $\mathrm{nm}$ UV light was proposed based on the analysis of the products. ${ }^{9}$ It is important to understand the water contamination process caused by quinolone antibacterial reagents. However, to our knowledge, there has been no report on the photochemical reaction of NAL in neat water. Since photochemical reaction are very fast and many products can be formed, it is generally used to postulate photochemical reaction pathways based on the product analysis. The aim of this study is to determine the products formed by the photochemical decomposition of NAL in neat water using $300 \mathrm{~nm}$ UV light, and to elucidate its photochemical decomposition pathway based on the product analysis.

\section{Experimental Section}

Reagents and general. Nalidixic acid (NAL) was purchased from the Sigma Chemical Co. (St. Louis, U.S.A.) and was used without further purification. The other chemicals were of reagent grade from the Aldrich Chemical Co. and were used as received. The aqueous NAL solution was saturated in quadruply distilled water, which was obtained by passing the distilled water through deionization system [Barnstead (U.S.A.) Nonopure II]. The concentration of the aqueous NAL solution was found to be $0.13 \mathrm{mM}$ by spectrophotometric method. The absorption spectra were taken using a UV-vis spectrophotometer (Uvikon, model 943, Italy). A deaerated NAL solution was prepared by bubbling $99.999 \%$ pure argon for approximately $60 \mathrm{~min}$.

Light source and actinometry. The irradiations were carried out at room temperature using a photochemical reactor (Rayonet, RPR-208) equipped with a UV lamp as a monochromatic light source $(\lambda=300 \mathrm{~nm})$. The intensity of the lamp irradiance was measured by potassium ferrioxalate actinometry. ${ }^{10,11}$ The reduction yield of $\mathrm{Fe}^{+3}$ (aq) to $\mathrm{Fe}^{+2}$ (aq) ion after irradiating the $6 \mathrm{mM}$ deaerated aqueous potassium ferrioxalate solution was measured by spectrophotometric method. ${ }^{12}$ The lamp intensity was estimated to be $7.66 \times$ $10^{18} \mathrm{~h} v \cdot \mathrm{mL}^{-1} \cdot \mathrm{min}^{-1}$ from the known quantum yield, Q $\left[\mathrm{Fe}^{+2}(\mathrm{aq})\right]=1 \cdot 24 .^{10,11}$ There was no change in the intensity of the lamp irradiance over the experimental period.

Isolation of the photochemical products. $60 \mathrm{~mL}$ of an irradiated aqueous NAL solution $\left(\leq 1.02 \times 10^{20} \mathrm{~h} v \cdot \mathrm{mL}^{-1}\right)$ was concentrated to approximately $5 \mathrm{~mL}$ using a rotary evaporator. The prepared sample was separated using liquid chromatography (Young-Lin, Model 930; Aqua $5 \mu \mathrm{C} 18$ column, eluent; $7: 3$ volume ratio of $20 \mathrm{mM}$ aqueous $\mathrm{KH}_{2} \mathrm{PO}_{4}$ solution : acetonitrile, UV detector) and four peaks were obtained. The four peaks were then isolated using preparative liquid chromatography (Shimadzu model LC8AD, Shim-Pack PREP-ODS (II) column, eluent; 30\% volume ratio of water : acetonitrilehe to $65 \%$ in $15 \mathrm{~min}$ ). Each isolated solution was then dried using a Freeze Dryer (Samwon, model SM 12).

Qualitative analysis of the photochemical products. The four isolated samples were analyzed using MALDITOF mass spectroscopy (Shimadzu, Kratos) and NMR spectroscopy (Varian Gemini). Nalidixic acid: $\mathrm{m} / \mathrm{z} 232,{ }^{1} \mathrm{H}-$ 
NMR $\left(\mathrm{CDCl}_{3}, 300 \mathrm{MHz}\right): \delta 1.45\left(3 \mathrm{H}, \mathrm{t}, \mathrm{N}(1) \mathrm{CH}_{2}-\mathrm{CH}_{3}\right)$, $2.64\left(3 \mathrm{H}, \mathrm{s}, \mathrm{N}(7)-\mathrm{CH}_{3}\right), 4.21\left(2 \mathrm{H}, \mathrm{q}, \mathrm{N}(1)-\mathrm{CH}_{2}\right), 7.19(1 \mathrm{H}, \mathrm{d}$, C(5)-H), 7.58 (1H, d, C(6)-H), $7.83(1 \mathrm{H}, \mathrm{s}, \mathrm{C}(2)-\mathrm{H}), 13.32$ $(1 \mathrm{H}$, s, C(3)-COO-H). 7-methyl-4-oxo-2,4-dihydro- $1 \mathrm{H}$ 8,8b-diaza-cyclobuta $[a]$ naphthalene-3-carboxylic acid (MCNC): $\mathrm{m} / \mathrm{z} 230,{ }^{1} \mathrm{H}-\mathrm{NMR}\left(\mathrm{CDCl}_{3}, 300 \mathrm{MHz}\right): \delta 2.69$ $\left(3 \mathrm{H}, \mathrm{s}, \mathrm{N}(7)-\mathrm{CH}_{3}\right), 3.93\left(2 \mathrm{H}, \mathrm{t}, \mathrm{N}(1)-\mathrm{CH}_{2}-\mathrm{CH}_{2}-\mathrm{C}(2), J=3.9\right.$ $\mathrm{Hz}), 4.93\left(2 \mathrm{H}, \mathrm{t}, \mathrm{N}(1)-\mathrm{CH}_{2}-\mathrm{CH}_{2}-\mathrm{C}(2), J=3.9 \mathrm{~Hz}\right), 7.34$ $(1 \mathrm{H}, \mathrm{d}, \mathrm{C}(5)-\mathrm{H}), 8.63(1 \mathrm{H}, \mathrm{d}, \mathrm{C}(6)-\mathrm{H}), 14.24(1 \mathrm{H}, \mathrm{s}, \mathrm{C}(3)-$ COO-H). 7-methyl-4-oxo-1,4-dihydro-[1,8]naphthyridine3-carboxylic acid (MDNC): $\mathrm{m} / \mathrm{z} 204,{ }^{1} \mathrm{H}-\mathrm{NMR}$ (DMSO- $d_{6}$, $300 \mathrm{MHz}): \delta 2.99\left(3 \mathrm{H}, \mathrm{s}, \mathrm{N}(7)-\mathrm{CH}_{3}\right), 7.5(1 \mathrm{H}, \mathrm{d}, \mathrm{C}(5)-\mathrm{H})$, $8.5(1 \mathrm{H}, \mathrm{d}, \mathrm{C}(6)-\mathrm{H}), 8.74(1 \mathrm{H}, \mathrm{s}, \mathrm{C}(2)-\mathrm{H}), 14.98(1 \mathrm{H}, \mathrm{s}$, C(3)-COO-H). 1,7-dimethyl-4-oxo-1,4-dihydro-[1,8]naphthyridine-3-carboxylic acid (DDNC): $\mathrm{m} / \mathrm{z}$ 218, ${ }^{1} \mathrm{H}-\mathrm{NMR}$ $\left(\mathrm{CDCl}_{3}, 300 \mathrm{MHz}\right): \delta 2.75\left(3 \mathrm{H}, \mathrm{s}, \mathrm{N}(7)-\mathrm{CH}_{3}\right), 4.09(3 \mathrm{H}, \mathrm{s}$, $\left.\mathrm{N}^{-C_{3}}{ }_{3}\right), 7.42(1 \mathrm{H}, \mathrm{d}, \mathrm{C}(5)-\mathrm{H}), 8.69(1 \mathrm{H}, \mathrm{d}, \mathrm{C}(6)-\mathrm{H}), 8.88$ $(1 \mathrm{H}, \mathrm{s}, \mathrm{C}(2)-\mathrm{H}), 14.98(1 \mathrm{H}, \mathrm{s}, \mathrm{C}(3)-\mathrm{COO}-\mathrm{H})$.

Quantitative analysis of the photochemical products. Quantitative analysis of the photochemical products was carried out by liquid chromatography (Young-Lin, Model 930; Aqua $5 \mu \mathrm{C} 18$ column, eluent; $7: 3$ volume ratio of 20 $\mathrm{mM}$ aqueous $\mathrm{KH}_{2} \mathrm{PO}_{4}$ solution:acetonitrile) with a UV detector $(\lambda=230 \mathrm{~nm})$. The amount of products formed as well as NAL decomposed was determined from a calibration curve using flumequine as the internal standard. Stock solutions of each product were prepared using the product isolated by the Freeze Dryer.

\section{Results and Discussion}

$0.13 \mathrm{mM}$ NAL in neat water, either aerated or deaerated, was irradiated with $300 \mathrm{~nm}$ UV light. The photochemical reaction was first examined by measuring the UV-vis absorption spectra before and after irradiating the solution. The UV-vis absorption spectrum of the NAL in neat water shows different absorption properties depending on the number of quanta. Figure 1 shows that the absorption spectra of NAL in neat water contain two bands, a strong peak at

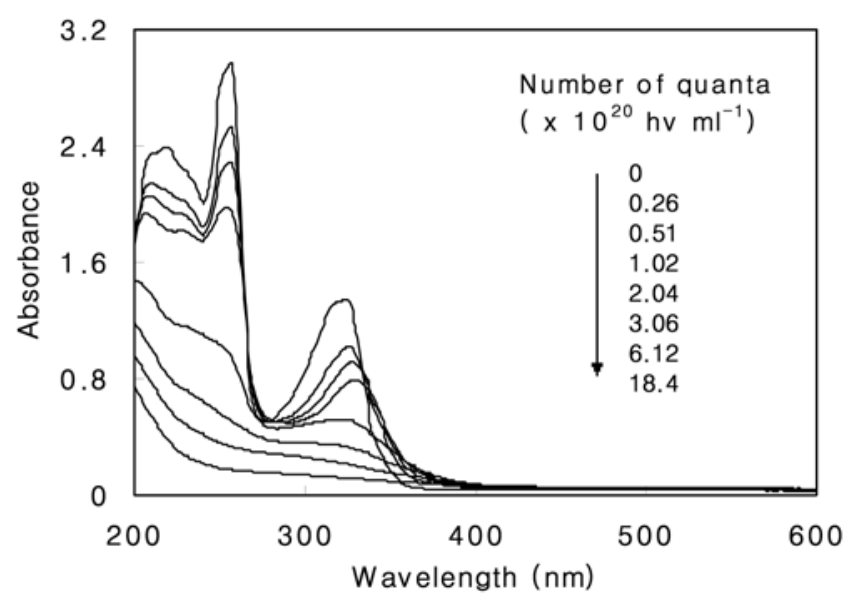

Figure 1. UV-vis absorption spectra before and after irradiation $(\lambda=300 \mathrm{~nm})$ of $0.13 \mathrm{mM}$ NAL in neat water.

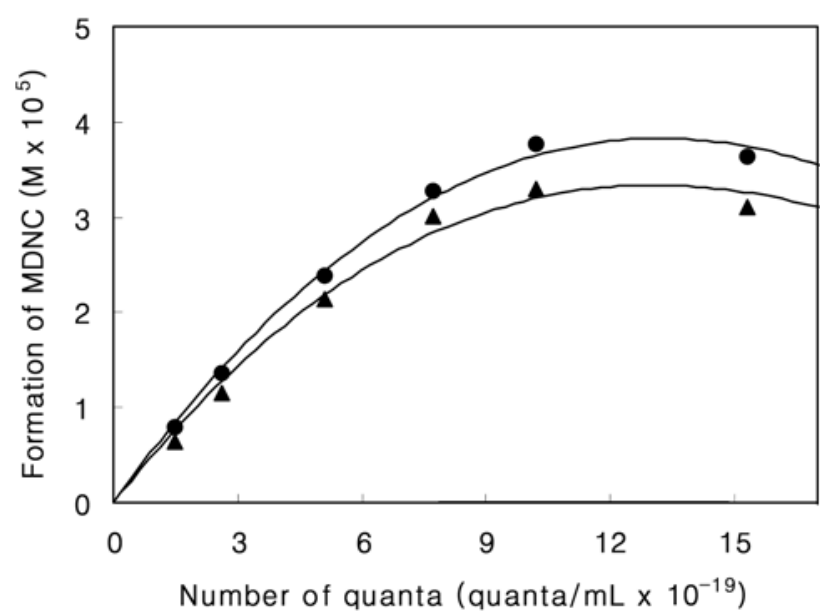

Figure 2. Formation of MDNC after irradiation of $0.13 \mathrm{mM}$ NAL in neat water as a function of the number of quanta: ( $\bullet$ ) in deaerated neat water; ( $\Delta$ ) in aerated neat water.

$258 \mathrm{~nm}$ and a weak peak at $331 \mathrm{~nm}$. The absorbance decreased and the absorption maxima of $331 \mathrm{~nm}$ shifted to a longer wavelength as the number of quanta increased. However, the absorption spectra had a similar shape up to a number of quanta of $1.02 \times 10^{20} \mathrm{~h} v \cdot \mathrm{mL}^{-1}$, whereas they were different when the number of quanta was above $2.04 \times$ $10^{20} \mathrm{~h} v \cdot \mathrm{L}^{-1}$. This means that the molecular structure of NAL is not so greatly altered in the initial state of the photochemical reaction. On the other hand, the shape of the absorption spectra appeared as a flat form when a number of quanta above $2.04 \times 10^{20} \mathrm{~h} v \cdot \mathrm{mL}^{-1}$ is applied. This suggests that aromatic moiety in NAL is broken down as the number of quanta is increased. During the irradiation of $0.13 \mathrm{mM}$ NAL in neat water, 7-methyl-4-oxo-2,4-dihydro- $1 H-8,8 \mathrm{~b}-$ diaza-cyclobuta $[a]$ naphthalene-3-carboxylic acid (MCNC), 7-methyl-4-oxo-1,4-dihydro-[1,8]naphthyridine-3-carboxylic acid (MDNC), and 1,7-dimethyl-4-oxo-1,4-dihydro-[1,8]naphthyridine-3-carboxylic acid (DDNC) were mainly produced in the initial state of the photochemical reaction.

The photolysis of $0.13 \mathrm{mM}$ NAL in deaerated and aerated neat water was studied as a function of the number of quanta. Figure 2 shows that in all cases, the formation of the products depends on the number of quanta. The tendency for the formation of products was not linearly proportional to the increase in the number of quanta, indicating that a back reaction, which contributes to the decomposition of the products, occurs in the system as a result of either the direct absorption of the UV light or by the attack of the radicals formed during the photochemical reaction. Therefore, the initial quantum yields $\left(\mathrm{Q}_{\mathrm{i}}\right)$ were determined from the slope of the tangent line of the curve in Figure 2. The results are summarized in Table 1.

After irradiating the NAL in neat water by $300 \mathrm{~nm} \mathrm{UV}$ light, only NAL absorbs the light and MCNC, MDNC and DDNC were mainly produced in the initial process of the reaction. These results are quite different from that for the methanolic NAL solution. Recently, it was reported that 1ethyl-7-methyl-4-oxo-4-hydro-1,8-naphthyridine (EMDN) 
Table 1. Initial quantum yields $\left(\mathrm{Q}_{\mathrm{i}}\right)$ of the compounds after irradiation $(\lambda=300 \mathrm{~nm})$ of $0.13 \mathrm{mM}$ nalidixic acid (NAL) in aerated and deaerated neat water

\begin{tabular}{ccc}
\hline \multirow{2}{*}{ Compounds } & \multicolumn{2}{c}{ Q $_{\mathrm{i}}$ when saturated with } \\
\cline { 2 - 3 } & $\mathrm{Ar}$ & Air \\
\hline $\mathrm{NAL}^{a}$ & $5.42 \times 10^{-3}$ & $2.76 \times 10^{-3}$ \\
MCNC $_{\text {MDNC }}$ & $3.73 \times 10^{-4}$ & $2.82 \times 10^{-4}$ \\
DDNC & $3.28 \times 10^{-4}$ & $2.56 \times 10^{-4}$ \\
\hline
\end{tabular}

${ }^{a}$ These values indicate the initial quantum yield of nalidixic acid decomposed. The others indicate the initial quantum yield of the products.

is formed as a major product in the irradiation of methanolic NAL solution. ${ }^{9}$ However, EMDN was not detected in the irradiation of NAL in neat water. This suggests that the photochemical reaction of NAL in neat water was different to that of the methanolic NAL solution. Furthermore, the spectroscopic properties such as the Stokes shift and the fluorescence quantum yield of NAL in neat water are quite different from those of the NAL in the methanol-water mixtures. ${ }^{9}$ Indeed, the electronically excited NAL in methanol has a chemical structure formed by the intramolecular hydrogen bond between the proton of the carboxyl group and the keto oxygen in NAL. The electronically excited NAL in neat water could more easily dissociated to the nalidixate anion form because the electronically excited state of aromatic compounds possesses larger dipole moment $\left(\mu^{*}\right)$ than that of the ground state $(\mu) .{ }^{13}$ Therefore, the electronically excited NAL in neat water has a chemical structure leading to a solvent-solute hydrogen bond as shown in Scheme 1 .

The hydrogen bond between the carboxylate anion and water molecules enhances the electronegativity of the carbon atom in position 2, and the electronically excited NAL is decomposed by splitting into radical $\mathbf{1}$ and a hydrogen atom as shown in Scheme 2. The formation of<smiles>CCn1cc(C(=O)OC(O)O)c(=O)c2ccc(C)nc21</smiles>

Scheme 1

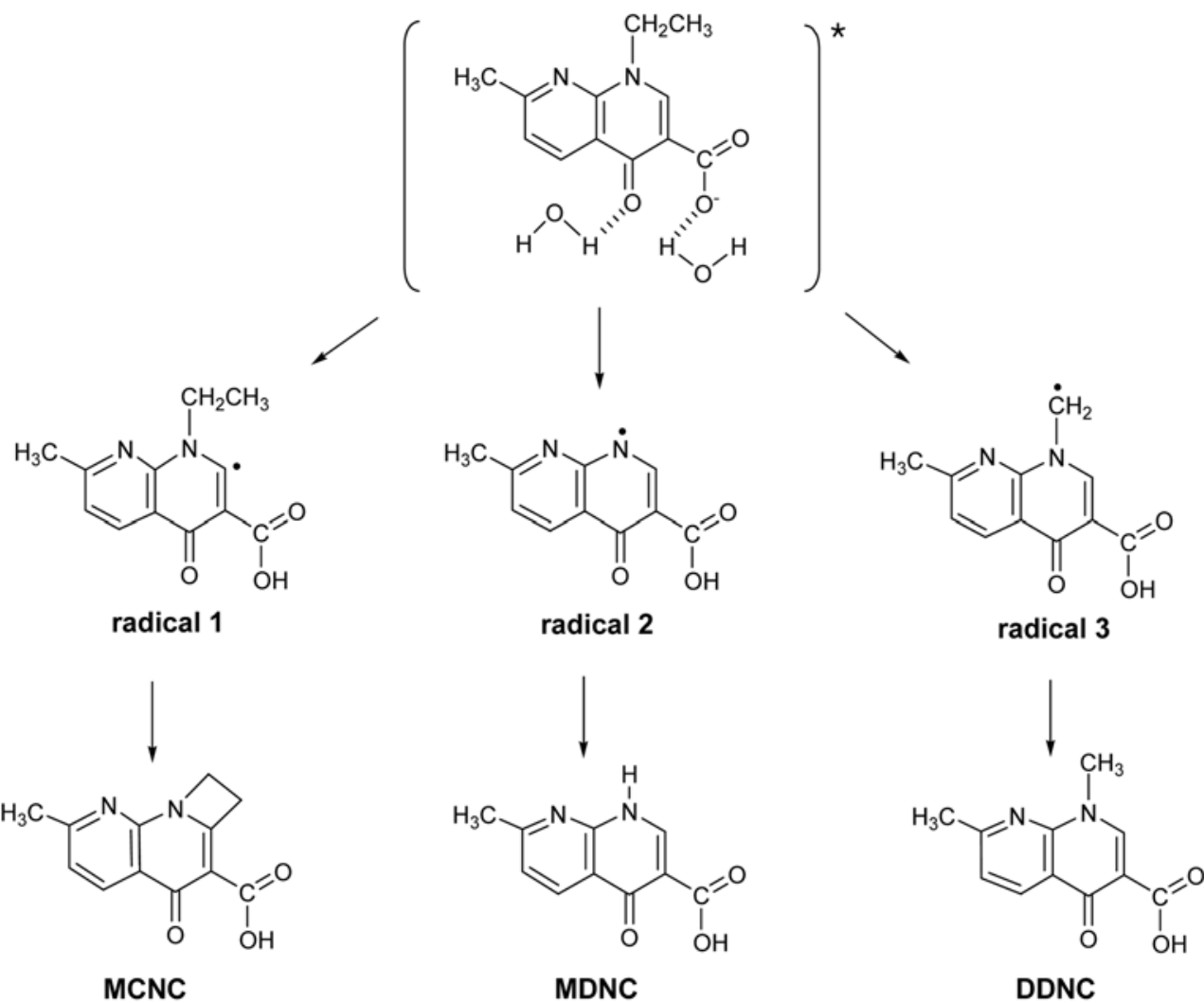

Scheme 2 
MCNC can be explained by the attachment of an ethyl group in position 1 to the radical 1 . The highly reactive species, hydrogen atom produced, can attack the other compounds in the solution. The fact that the formation of the products is not linearly proportional to the increment in the number of quanta, supports that this hypothesis is reasonable. In addition, the hydrogen bond between the keto oxygen and water molecules might also effect the electronegativity of the nitrogen atom in position 1 through resonance with the delocalized $\pi$ electron in naphthyridine ring of the NAL molecule. The enhanced electronegativity of the nitrogen atom could weaken the bond between the nitrogen atom in position 1 and the ethyl group leading to radical $\mathbf{2}$ as well as a $\mathrm{C}-\mathrm{C}$ bond in the ethyl group leading to radical 3 . The radicals attack the other compounds in the solution, and are converted to MDNC and DDNC.

As shown in Table 1, the initial quantum yield $\left(Q_{i}\right)$ of the NAL decomposed in the deaerated neat water was greater than that in the aerated neat water. Furthermore, less MCNC, MDNC and DDNC were produced during the initial process of the reaction after irradiating the aerated neat water than the deaerated neat water. This means that oxygen affects the photolysis of NAL in neat water. It is known that oxygen is a good dynamic quencher, and the electronically excited NAL can transfer its energy to oxygen very fast in air atmosphere. As a result, there might be a lower population of electronically excited NAL and a lower amount of decomposed NAL.

In conclusion, the electronically excited NAL in neat water after the absorption of $300 \mathrm{~nm}$ UV light exists as its dissociated form, and a solvent-solute hydrogen bonding is formed in the solution. In the initial process of irradiation, 7methyl-4-oxo-2,4-dihydro- $1 H$-8,8b-diaza-cyclobuta $[a]$ naphthalene-3-carboxylic acid (MCNC), 7-methyl-4-oxo-1,4dihydro-[1,8]naphthyridine-3-carboxylic acid (MDNC), and 1,7-dimethyl-4-oxo-1,4-dihydro-[1,8]naphthyridine-3-carboxylic acid (DDNC) were produced as the major products during the irradiation of $0.13 \mathrm{mM}$ NAL in neat water. The products were also decomposed with increasing number of quanta. The photochemical reaction of NAL was affected by the presence of oxygen in the solution. The decomposition of NAL as well as the formation of MCNC, MDNC and DDNC was faster in the deaerated neat water than in the aerated neat water.

Acknowledgment. This study was financially supported by special research fund of Chonnam National University in 2004.

\section{References}

1. Lesher, G.; Froelich, M.; Gruett, J. J. Med. Pharm. Chem. 1962, 5, 1063.

2. Kang, J. S.; Kim, T. H.; Park, K. B.; Chung, B. H.; Youn, J. I. Photodermal. Photoimmunol. Photome. 1993, 9, 159.

3. Cho, Y. H.; Kim, T. H.; Park, H. B.; Park, C. K.; Park, K. M. Korean J. Dermatol. 1995, 33, 1021.

4. Ferguson, J. Photochem. and Photobiol. 1995, 62, 954.

5. Detzer, N.; Huber, B. Tetrahedron 1975, 31, 1937.

6. Moore, D. E.; Hemmens, V. J.; Yip, H. Photochem. Photobiol. 1984, 39, 57.

7. Vermeersch, G.; Ronfard-Haret, J. C.; Bazin, M.; Carillet, V.; Moriere, P.; Santus, R. Photochem. and Photobiol. 1991, 54, 661.

8. Fernandez, E.; Cardenas, A. M. J. Photochem. Photobiol., B: Photobiol. 1990, 4, 329.

9. Park, H. R.; Park, O. H.; Lee, H. Y.; Seo, J. J.; Bark, K. M. Bull. Korean Chem. Soc. 2003, 24, 1618.

10. Hatchard, C. G.; Parker, C. A. Proc. Roy. Soc. (London) 1956, A235, 518.

11. Lee, J.; Seliger, H. H. J. Chem. Phys. 1968, 40, 519.

12. Atkins, R. C. J. Chem. Ed. 1975, 52, 550.

13. Lakowicz, J. R. Principles of Fluorescence Spectroscopy; Plenum Press: New York, USA, 1983; p 188. 